Results Infarct size was slightly reduced in AVE 0991 group compared to control group ( $42.6 \pm 3.6 \%$ vs $50.9 \pm 4.4 \%, \mathrm{p}<0.01)$. In addition, AVE 0991 reduced MI-induced hypertrophy as quantified by diameter measurements of cardiomyocytes (vs. control group $25.49 \pm 4.37 \mu \mathrm{m}$ vs $32.06 \pm 6.85 \mu \mathrm{m}, \mathrm{p}<0.05)$. The overexpression of TGF- $\beta 1$ and TNF- $\alpha$ mRNA were inhibited by chronic AVE 0991 treatment alone (vs. control group, TGF- $\beta 1$ : $4.15 \pm 1.18$ vs $14.23 \pm 3.84, \quad \mathrm{p}<0.05$; TNF- $\alpha: 2.21 \pm 0.44$ vs $3.87 \pm 0.55, \mathrm{p}<0.05$, respectively). AVE 0991 markedly attenuated the increase of the expression of Collagen I $(1.79 \pm 0.15$ vs $4.3 \pm 0.75, \mathrm{p}<0.001)$ and III $(3.12 \pm 0.42$ vs $9.55 \pm 0.83, \mathrm{p}<0.001)$ mRNA compared to control group.

Conclusion The nonpeptide angiotensin-(1-7) analogue AVE 0991 could attenuate overexpression of inflammatory cytokines and ventricular remodelling induced by myocardial infarction (MI).

\section{e0094 RESEARCH OF TRIPTOLIDE ON ANG II-INDUCED NEONATAL CARDIAC FIBROBLASTS PROLIFERATION AND COLLAGEN SYNTHESIS}

doi:10.1136/hrt.2010.208967.94

${ }^{1}$ Zhang Kai, ${ }^{1}$ Hasimu Buaijiaer, ${ }^{1}$ Yuan Binbin, ${ }^{2}$ Zhang Zhengang. ${ }^{1}$ Department of Cardiology, Nanjing Benq Hospital, The Affiliated Hospital of Nanjing Medical University; ${ }^{2}$ Department of Cardiology of The First Peoples Hospital of Yangzhou University

Objectives To observe whether TP can inhibit proliferation and collagen synthesis of cardiac fibroblasts and clarify the possible mechanisms.

Methods Differential attachment technique to obtain and cultivate neonatal SD rat cardiac fibroblasts. Experimental cells were randomly divided into five groups: (1) control (culture medium); (2) Ang II group (10-7 mol/1 Ang II); (3) $1 \mu \mathrm{g} / 1 \mathrm{TP}$ group $(1 \mu \mathrm{g} / 1 \mathrm{TP}+10-$ $7 \mathrm{~mol} / \mathrm{l}$ Ang II); (4) $10 \mu \mathrm{g} / \mathrm{l}$ TP group $(10 \mu \mathrm{g} / \mathrm{l} \mathrm{TP}+10-7 \mathrm{~mol} / \mathrm{l}$ Ang II); (5) $100 \mu \mathrm{g} / 1 \mathrm{TP}$ group $(100 \mu \mathrm{g} / 1 \mathrm{TP}+10-7 \mathrm{~mol} / \mathrm{l}$ Ang II). Ang II are simultaneously added into the culture medium. MTT colorimetric determination of cell proliferation. Collagen synthesis, TGF $\beta 1$ secretion and phosphorylation of extracellular signal-regulated kinase 1/2 (ERK1/2) by Hydroxyproline, ELISA and Wertern Bloting.

Results 1 . The proliferation of $\mathrm{CFb}$ is significantly promoted after adding Ang II, compared with the control group $(\mathrm{p}<0.05$ or $\mathrm{p}<0.01)$. The $100 \mu \mathrm{g} / 1 \mathrm{TP}$ showed the effect of inhibiting the proliferation of $\mathrm{CFb}$ at the first $24 \mathrm{~h}(\mathrm{p}<0.01)$, reached a peak within $48 \mathrm{~h}$ $(\mathrm{p}<0.001)$, started to diminish after $72 \mathrm{~h}$, indicate the best time to exert effects were at 2 to 3 days. 2. With the time increase after adding Ang II, collagen synthesis increased, there is significant difference compared with the control group $(p<0.05$ or $p<0.01)$. After $24 \mathrm{~h}, 48 \mathrm{~h}, 72 \mathrm{~h}$ of adding TP, the collagen content of each group compared with the Ang II group were significantly different. The effect of high concentration TP $(100 \mu \mathrm{g} / \mathrm{l})$ reached the peak $(p<0.001)$ at $48 \mathrm{~h}(\mathrm{p}<0.001)$. 3. After $24 \mathrm{~h}$ of adding Ang II, TGF- $\beta_{1}$ expression was significantly increased $(p<0.01)$. After $24 \mathrm{~h}$ of adding different concentrations of TP, TGF- $\beta_{1}$ expression were significantly decreased $(p<0.05$ or $p<0.01)$. 4. After $30 \mathrm{~min}$ of adding Ang II, ERK1/2 phosphorylation increased compared with the negative control $(p<0.05)$. After $30 \mathrm{~min}$ of adding $100 \mu \mathrm{g} / \mathrm{l}$ TP, $\mathrm{p}-\mathrm{ERK} 1 / 2$ expression decreased compared with the Ang II group $(p<0.05)$. And $1 \mu \mathrm{g} / 1,10 \mu \mathrm{g} / 1 \mathrm{TP}$ did not inhibit ERK1/2 phosphorylation caused by Ang II. Positive control U0126 significantly inhibited the ERK1/2 phosphorylation $(\mathrm{p}<0.01)$.

Conclusions Ang II promote neonatal SD rat cardiac fibroblasts proliferation and collagen synthesis, the possible mechanism may be the MAPK signal transduction pathway. Ang II has the effect of promoting cardiac fibroblasts proliferation by increase phosphorylation of ERK1/2 expression; promoting collagen synthesis by increasing the expression of TGF- $\beta_{1}$. Triptolide significantly inhibited the Ang II-induced cardiac fibroblast proliferation and collagen synthesis in a dose-dependent manner, and the mechanism is probably by inhibiting the ERK1/2 phosphorylation and reducing the expression of TGF- $\beta_{1}$.

\section{e0095 THE STUDY ON THE RELATIONS BETWEEN RENNIN- ANGIOTENSIN-ALDOSTERONE SYSTEM AND ATRIAL STRUCTURAL REMODELLING AND ATRIAL FIBRILLATION}

doi:10.1136/hrt.2010.208967.95

Xiaohui Liu, Taiyang Luo, Tao Lei, Xingpeng Liu, Haiyun Wang, Changsheng Ma. Beijing Anzhen Hospital, Capital Medical University

Objective To investigate the effects of perindopril and/or spironolactone on atrial structural and functional remodelling in atrial fibrillation (AF) dogs induced by chronic rapid atrial pacing, and research the relations between rennin-angioensin-aldosterone system (RAAS) and atrial interstitial remodelling and atrial fibrillation.

Methods 24 healthy male hybrid dogs aged 15-18 months were paced for 8 weeks and randomly divided into four groups: control group, perindopril group $(\mathrm{P})$, spironolactone group $(\mathrm{S})$, and combination of perindopril and spironolactone group $(\mathrm{P}+\mathrm{S})$. The dogs in $\mathrm{P}$ group, $\mathrm{S}$ group, and $\mathrm{P}+\mathrm{S}$ group respectively received perindopril $\left(1 \mathrm{mg} \cdot \mathrm{kg}^{-1} \cdot \mathrm{d}^{-1}\right)$ and/or spironolactone $\left(10 \mathrm{mg} \cdot \mathrm{kg}^{-1} \cdot \mathrm{d}^{-1}\right)$. Plasma Angiotensin II (Ang II) and aldosterone (Ald) were measured before and after 4 and 8 weeks pacing. Transthoracic echocardiographic examinations were performed before and after 8 weeks pacing. The number of dogs maintained AF and duration of AF after stopping of pacing were recorded. Atrial collagen volume fraction (CVF) was analysed by Masson staining after 8 weeks pacing.

Results (1) Plasma Ang II and Ald were no significant differences between four groups before pacing. Compared with the control group, plasma Ang II and Ald after 4 and 8 weeks pacing in P group, $\mathrm{S}$ group and $\mathrm{P}+\mathrm{S}$ group were significantly lower. In the control group, plasma Ang II and Ald levels after 4 and 8 weeks pacing was significantly higher than that before pacing; in the other groups, there were no significant differences. (2) Compared with the control group, the diameter, end-systolic volume and end-diastolic volume of the left atrium of $\mathrm{P}$ group, $\mathrm{S}$ group and $\mathrm{P}+\mathrm{S}$ group after pacing significantly reduced, but LAEF significantly increased after 8 weeks pacing. (3) Compared with the control group, the rate of dogs maintained atrial fibrillation of three drug treatment groups after stopping of pacing significantly reduced, with a shorter average duration of AF. (4) Compared with the control group, the value of $\mathrm{CVF}$ in $\mathrm{P}$ group, $\mathrm{S}$ group and $\mathrm{P}+\mathrm{S}$ group was significantly lower.

Conclusion The occurrence and development of atrial fibrillation and atrial structural remodelling is closely related to RAAS activation. The RASS blockers can inhibit atrial fibrosis, improve the changes of atrial structure and function, and reduce the incidence and duration of atrial fibrillation in the atrial fibrillation dogs induced by chronic rapid atrial pacing.

\section{e0096 THE STUDY ON THE RELATIONS BETWEEN ALDOSTERONE AND ATRIAL INTERSTITIAL REMODELLING AND ATRIAL FIBRILLATION}

doi:10.1136/hrt.2010.208967.96

Taiyang Luo, Xiaohui Liu, Xin Du, Xingpeng Liu, Tao Lei, Haiyun Wang, Jiahai Shi. Beijing Anzhen Hospital, Capital Medical University

Objective To investigate the relations between aldosterone (Ald) and atrial interstitial remodelling and atrial fibrillation (AF). 